\title{
Consideraciones restauradoras y periodontales de molares tratados con resección radicular
} Restoration and periodontal considerations of resectioned molar teeth

\author{
CUARTAS RAMÍREZ JC* \\ ARDILA MEDINA CM**
}

Cuartas Ramírez JC, Ardila Medina CM. Consideraciones restauradoras y periodontales de molares tratados con resección radicular. Av Periodon Implantol. 2010; 22, 3: 157-164.

\begin{abstract}
RESUMEN
La restauración de molares sometidos a resección radicular debe realizarse cuidadosa y apropiadamente. Los problemas que pueden aparecer cuando la terapia restauradora no es llevada a cabo adecuadamente incluyen problemas de retención, excesiva debilidad de la estructura radicular e incompleto sellado marginal. La cirugía preprotésica es una fase importante de este proceso y aun cuando la reparación tisular ocurre 6 u 8 semanas después del corte, se pueden evaluar diferentes tipos de restauraciones de acuerdo al entorno periodontal, sin embargo, su pronóstico a largo plazo es incierto. Los estudios clínicos indican que los principales fracasos son de origen periodontal, endodóntico y biomecánico.
\end{abstract}

PALABRAS CLAVE: Terapia radicular, compromiso de furca, restauración.

\section{SUIMIMARY}

Great care should be taken in properly restoring molars that have undergone root resection surgery. Problems that can arise when reconstructive treatment is not correctly carried out comprise faulty retention, excessive weakening of root structure, incomplete marginal seal. Preprothesic surgery is a particularly important step of the process and although tissue repair occurs 6 to 8 weeks after coronoradicular resection, different types of prostheses can be evaluated according to the periodontal environment. The long-term prognosis for this type of restoration is uncertain. Clinical studies indicate that the main sources of failure are periodontal, endodontic, and biomechanical.

KEY WORDS: Root therapy, furcation involvement, restoration.

Fecha de recepción: 15 de enero de 2009.

Fecha de aceptación: 30 de enero de 2009.

\section{INTRODUCCIÓN}

La morfología de las furcas hace que su tratamiento efectivo se convierta en un reto importante para la terapia periodontal, y como consecuencia, se han propuesto tratamientos específicos para enfrentar tal desafío. La selección del tratamiento apropiado para una situación particular depende de diferentes factores que deben ser cuidadosamente evaluados antes de iniciarlo: grado de compromiso de la bifurcación, pro- porción corona raíz, longitud de las raíces, morfología radicular, valor estratégico del diente, movilidad dentaria residual, requerimientos protésicos, condición periodontal de los dientes adyacentes, habilidad para mantener una adecuada higiene bucal $(1,2)$. La tabla 1 presenta algunas opciones de tratamientos para manejar los compromisos de furca. Una de las posibilidades terapéuticas es la resección radicular ( $R R)$, definida por la Academia Americana de Periodoncia como la remoción quirúrgica de una o más raíces de un dien- 


\section{TABLA 1.- OPCIONES DE TRATAMIENTO PARA MANEJAR LOS COIMPROMISOS DE FURCA}

- Preparación radicular a campo abierto y a campo cerrado

- Odontoplastia

- Procedimientos de tunelización

- Corte corono-radicular

- Bicuspidización

- Enfoques regenerativos

Exodoncia/implante te multirradicular incluyendo o no su área coronal correspondiente (3). Es importante aclarar que los términos amputación radicular y hemisección también son reconocidos conjuntamente por algunos autores con el nombre de $R R(4,5)$. Este procedimiento hace posible mantener dientes que estaban predestinados para exodoncia (6-9) y debe seguirse por una restauración de la parte del diente que se ha conservado, la cual debe preservar adecuadamente las condiciones periodontales y los principios restauradores (6). El pronóstico a largo plazo de este tipo de restauraciones es incierto y sus posibles fracasos pueden ser de origen periodontal, endodóntico y biomecánico (1013). Aun cuando el advenimiento de los implantes oseointegrados ha conducido a declinar el empleo de procedimientos radiculares resectivos, estos pueden convertirse en una alternativa efectiva en algunas situaciones, además, la disponibilidad de implantes no justifica la remoción de dientes naturales que de alguna manera pueden igualmente proporcionar buena función. El objetivo de este artículo es presentar las consideraciones restauradoras y periodontales que se deben tener en cuenta en los molares sometidos a RR y su pronóstico.

\section{CONSIDERACIONES RESTAURADORAS}

Se deben considerar algunos factores en la restauración de molares con RR como resultado de las características anatómicas específicas que ocasiona este procedimiento. Debido a que las raíces de los molares son más estrechas mesiodistalmente y más amplias bucolingualmente que la mayoría de los dientes unirradiculares $(14,15)$, se requieren algunas modificaciones en el diseño de la preparación protésica. Es fundamental un perfil de emergencia plano del margen de la preparación para establecer un entorno que permita un adecuado control de placa bacteriana. Debido a la pérdida de estructura dental originada por el procedimiento de RR es necesario reconstruir la parte coronal remanente con un material adhesivo tipo resina, ya que algunos autores no recomiendan la incorporación de postes colados por que podrían aumentar la posibilidad de fractura radicular $(16,17)$. Sin embargo, está indicado un poste prefabricado en aquellas situaciones donde la estructura coronal es insuficiente para proporcionar una adecuada retención y resistencia cuando se va a realizar una corona completa. Relacionado con este último tópico, Torbjorner y colaboradores (18), estudiaron 638 pacientes tratados con 788 postes colados y para-postes durante cinco años. La tasa de fracaso acumulada fue del $8 \%$ para el grupo conformado por 332 para-postes y del $15 \%$ para 456 postes colados, diferencias que fueron estadísticamente significativas $(p<0,05)$. Por el contrario, una revisión sistemática realizada por Heydecke y colaboradores (19), concluyó que no existía diferencia en el comportamiento de fractura en aquellos dientes restaurados con diferentes clases de postes, resultados que han sido corroborados por otros investigadores (20-22).

El tipo de margen de la corona completa es otro aspecto importante. Debido a la limitada amplitud residual, se recomiendan terminaciones en filo de cuchillo para evitar la remoción excesiva de estructura dentaria $(2,7)$. En el área cervical, esta línea de terminación es muy trascendental, cuando el clínico desea obtener un efecto férula $(2,9)$.

Finalmente, para disminuir las cargas oclusales excesivas es fundamental la reducción de las inclinaciones cuspídeas de la restauración $(23,24)$.

\section{CONSIDERACIONES PERIODONTALES}

Asumiendo una distancia de $2 \mathrm{~mm}$ para la amplitud biológica y 1,5 $\mathrm{mm}$ para la estructura dentaria requerida para lograr un efecto férula en la corona, indica que se requiere un mínimo de 3,5 mm disponibles entre la cámara pulpar y el margen coronal de las raíces residuales $(2,7,9)$. Deutchs y colaboradores (25), evaluaron 200 molares empleando un estéreo-microscopio, demostrando una distancia del piso de la cámara pulpar a la furca de $3,05 \pm 0,79$ y $2,96 \pm 0,78$ en los molares superiores e inferiores respectivamente. De la misma forma, en una investigación in vitro, se evaluaron 50 molares superiores sometidos a resección 
radicular de la raíz distovestibular (26). Los autores informaron que en el $86 \%$ de los dientes medidos, se presentó una estructura dentaria disponible menor a $3 \mathrm{~mm}$. A partir de estos datos, se puede inferir entonces, que las restauraciones realizadas se extienden subgingivalmente. Esto es de particular importancia en aquellos casos en donde la RR de molares superiores establece una amplitud biológica que necesariamente conduce a la invasión de la furca entre las dos raíces residuales $(2,7)$.

Un aspecto fundamental en los procedimientos de RR es el contorneado óseo con el fin de recrear una arquitectura positiva en donde los colgajos deben ser posicionados apicalmente, con el fin de obtener un entorno adecuado para una buena higiene bucal (2527). La presencia de remanentes de las raíces (labios radiculares) sin detectar durante el procedimiento quirúrgico debe ser eliminada durante la fase restauradora ya que el mantenimiento de tal morfología puede ocasionar acumulación de placa y progreso de la enfermedad periodontal (2, 9). En un estudio donde se examinó la calidad de las RR de 70 molares en 62 pacientes se encontró que 21 de las resecciones (30\%) mostraron fracasos relacionados con remanentes radiculares residuales y presencia de labios relacionados con la furca (28). Los fracasos fueron más frecuentes en los molares superiores $(33,3 \%)$ que en los inferiores $(22,7 \%)$ debido principalmente a que los primeros fueron observados solamente en el 37,5\% de las radiografías.

En la figura 1 se presenta un molar superior con un compromiso endoperiodontal que afectó la raíz me-

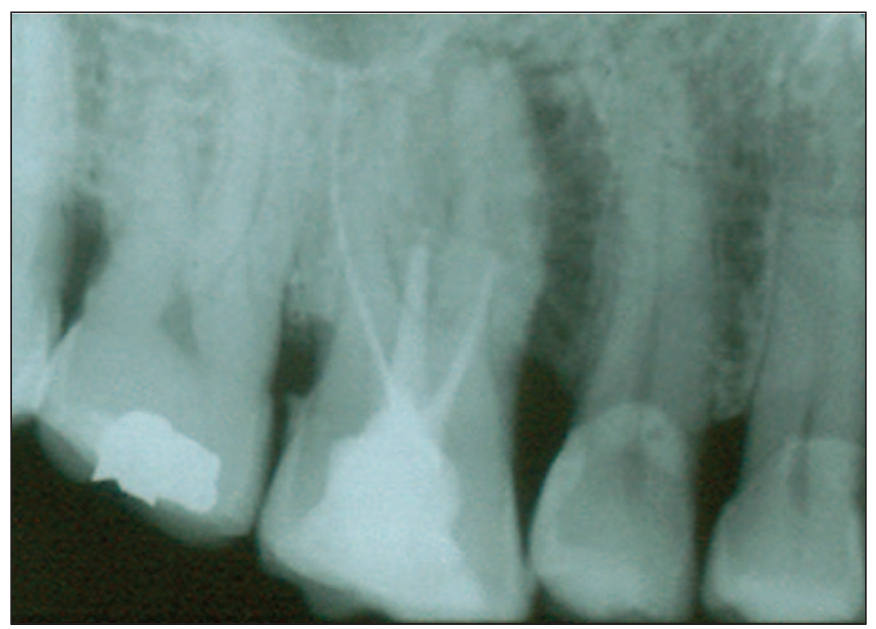

Fig. 1: Primer molar superior con compromiso endoperiodontal. sial y la bifurcación mesiovestibular. La figura 2 muestra el mismo molar tratado con $\mathrm{RR}$, retratamiento de los conductos radiculares y restauración con corona en metal porcelana. Las figuras 3 y 4 presentan una vista mesial y cervical respectivamente de la restauración, donde se aprecia el contorno y perfil de emergencia que respetan la nueva morfología radicular.

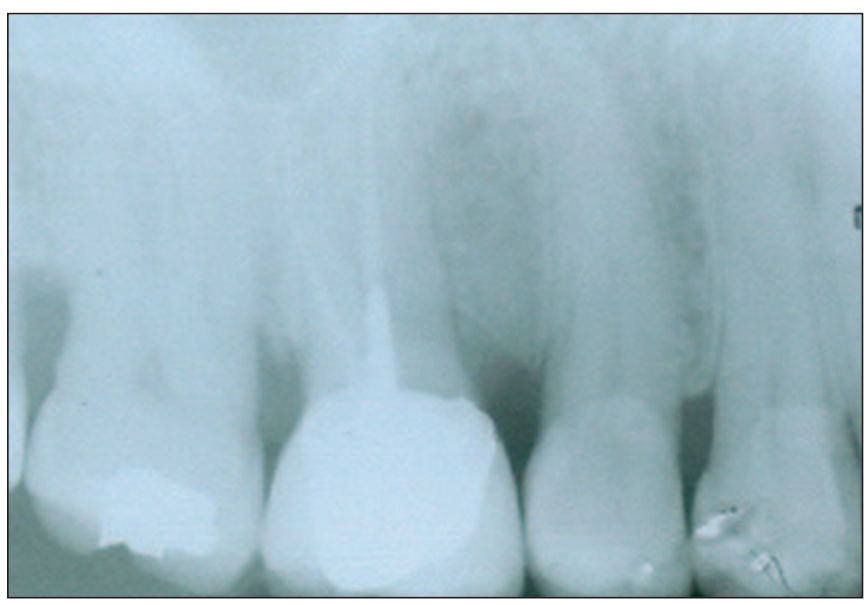

Fig. 2: Tratamiento del primer molar superior con resección radicular y corona metal porcelana.

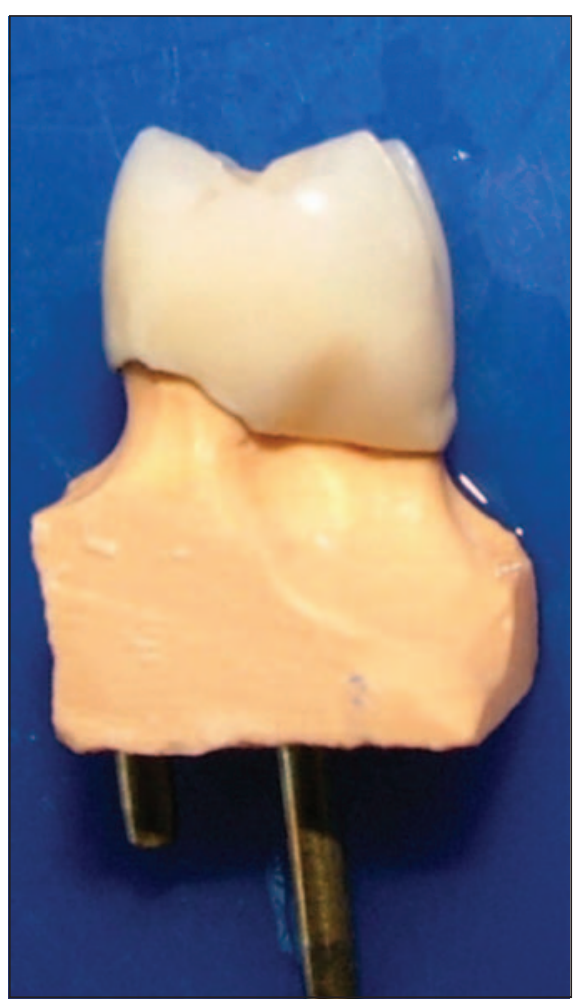

Fig. 3: Vista mesial de la restauración. 


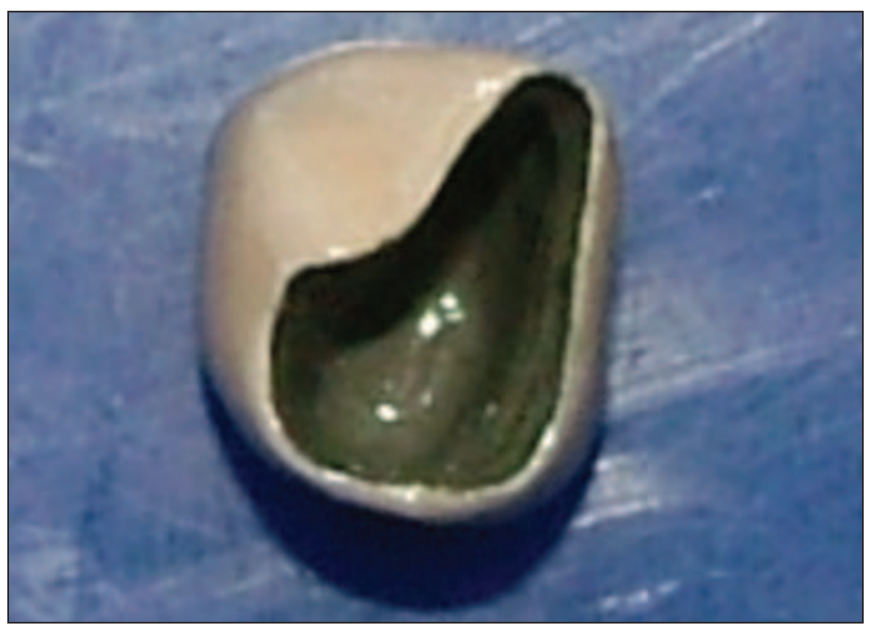

Fig. 4: Vista cervical de la restauración.

\section{PRONÓSTICO DE LA RESECCIÓN RADICULAR}

Una gran cantidad de investigadores han evaluado la efectividad y el pronóstico de la RR en el tratamiento de los molares con compromisos de furca. La mayoría de ellos están de acuerdo en que estos procedimientos presentan un alto grado de complejidad y requieren de grandes destrezas quirúrgicas y restauradoras. Bergenholtz (29), realizó un estudio longitudinal donde examinó 45 dientes tratados con $R R$, de los cuales evaluó 21 de ellos después de 2 a 5 años y 17 posterior a un período comprendido entre 5 y 10 años. Solamente tres molares $(6 \%)$ fueron extraídos: dos por razones periodontales y uno por complicaciones endodónticas.

Se evaluaron 34 molares superiores durante un periodo entre 11 y 84 meses posteriores a la RR (30). El hallazgo más notorio de esta investigación fue que solamente tres de los molares presentaron movilidad, de los cuales dos, fueron pilares de dentaduras removibles. Solamente uno de los molares fue extraído después de tres años debido a abscesos laterales recurrentes y posterior compromiso de la furca mesial.

En una investigación se examinaron 100 sujetos con 310 dientes multirradiculares sometidos a diferentes tratamientos periodontales (31), 135 fueron extraídos durante la fase inicial y 87 fueron seleccionados para RR. Ninguno de los molares se perdió durante el período de observación.

Blomlöf y colaboradores (32), realizaron un estudio en 246 dientes con el fin de comparar la mortalidad en
146 molares con $R R$ y 100 dientes unirradiculares por un período de 3 a 10 años (promedio 5.8 años). La tasa de supervivencia fue del $68 \%$ y del $77 \%$ respectivamente y la diferencia no fue estadísticamente significativa. En conclusión, el pronóstico de la RR no es más pobre que el de los dientes unirradiculares con igual susceptibilidad a la periodontitis, si son óptimas las condiciones endodónticas y el mantenimiento.

Se examinaron clínica y radiográficamente 34 molares ( 7 superiores y 27 inferiores) con $R R$ durante un periodo comprendido entre 1 y 7 años (promedio 2,9 años) (33). Todos los pacientes fueron llamados para mantenimiento cada 3-6 meses. Las indicaciones para la RR fueron periodontales ( 9 casos), endodónticas (20 casos), caries (4 casos) y un granuloma. 22 de los dientes tratados fueron usados como pilares distales para prótesis fijas y los restantes fueron restaurados con coronas completas individuales. De los 34 molares tratados, seis fallaron por razones endodónticas y solamente uno por motivos periodontales. No se encontró correlación entre la falla y la longitud del período de seguimiento. Tampoco se halló diferencia en la profundidad del surco gingival o en la altura de la cresta alveolar al comparar dientes sometidos o no a RR.

Se realizó un estudio retrospectivo con el fin de investigar las diferencias en los parámetros clínicos periodontales entre molares pilares con y sin $R R$ antes y después de la terapia periodontal y protésica, usando coronas telescópicas (25). Se trataron un total de 85 molares (47 superiores y 38 inferiores) fueron tratados en 25 sujetos. De los 85 pilares 52 presentaron RR y todos fueron observados durante un período promedio de $6,7 \pm 1,9$ años (rango: 5-13 años). Se evaluaron las diferencias en los índices de placa (34), índice gingival (35), profundidad de sondaje, nivel de inserción y cambios en el hueso alveolar, antes y después de la terapia periodontal y protésica en los dientes sometidos o no a RR. Los resultados mostraron una gran mejoría de los índices periodontales en los molares con compromiso de furca grado II y III que fueron sometidos a RR y restaurados con coronas telescópicas comparados con aquellos sin RR.

En una investigación se evaluaron 32 pacientes con 49 $R R$ que estuvieron bajo mantenimiento regular (3-6 meses) durante 11 años y medio (rango: 2-23 años) (36). Las modalidades de tratamiento fueron similares para todos los pacientes. La terapia endodóntica se realizó conservadoramente y previo a la RR. Se emplearon algunos postes colados y las restauraciones provisionales se realizaron antes del tratamiento periodontal. La mayoría de pacientes fueron tratados con coronas com- 
pletas individuales y prótesis fijas. El $92 \%$ de todos los dientes con RR permanecieron en boca 12 años aproximadamente y los fracasos fueron causados por caries y razones endodónticas.

Carnevale (11), diseñó una investigación para evaluar el efecto a largo plazo de molares con RR. La muestra incluyó 72 sujetos con edades comprendidas entre los 21 y 62 años de edad, empleando 175 molares control y 175 molares con RR. Los pacientes fueron reexaminados a los 3, 5 y 10 años postoperatorios. Los resultados de la evaluación presentaron una tasa de supervivencia de $93 \%$ para los dientes con RR y del $99 \%$ para los molares control, después de 10 años de seguimiento.

Los resultados positivos de los procedimientos de $R R$ informados por diferentes investigadores fueron atribuidos al restablecimiento de una morfología radicular favorable, restauraciones con perfiles de emergencia apropiados, estrictos controles de placa bacteriana y establecimiento de un programa regular de mantenimiento $(11,29-33,36)$.

Por otra parte, resultados menos favorables se han publicado en otros estudios. Langer y colaboradores (37), evaluaron retrospectivamente la tasa de éxito de 100 molares con RR (50 superiores y 50 inferiores). Después de cuatro años se perdieron solamente el $6 \%$ de los dientes, incrementándose a 15,8\% en el quinto año y a $45 \%$ después de 10 años. Los fracasos fueron mayores en los molares superiores y se presentaron por causas periodontales (10 dientes, $26,3 \%$ ), fracturas radiculares (25 dientes, $47,4 \%$ ), fracasos endodónticos (7 dientes, 18,4\%) y fallos en la cementación de las coronas completas (3 dientes, 7,9\%).

Bühler (38), evaluó 28 molares con RR usados como pilares de prótesis fija. Los resultados no mostraron ningún fracaso en los primeros cuatro años, 10,7\% (3 dientes) fallaron en el periodo comprendido entre 5-7 años y $32,1 \%$ (9 dientes) fracasaron después de 10 años.

Es evidente la variabilidad en las tasas de éxito presentadas por los autores, causadas posiblemente por criterios de inclusión diferentes y por la presencia de factores técnicos importantes como los métodos de restauración de los dientes con RR. Con el fin de mejorar la posibilidad de comparación de los estudios se realizó un metaanálisis para intentar reducir los resultados a denominadores comunes de tiempos de observación y criterios de fracaso (39). Empleando estos supuestos, se demostró que en un período de observación de siete años, la tasa de fracaso para los dientes tratados con RR fue de 13,1\%. Otro dato importante que arrojó esta publicación fue que tales tasas de éxito son comparables con las de los implantes endoóseos ubicados en zonas posteriores. La tabla 2 presenta las tasas de fracaso de la terapia de RR demostradas por algunos investigadores.

TABLA 2.- TASAS DE FRACASO DE LA RESECCIÓN RADICULAR

\begin{tabular}{|l|c|c|c|c|c|c|c|c|}
\hline $\begin{array}{l}\text { Tiempo de } \\
\text { observación }\end{array}$ & $\begin{array}{c}\text { Número } \\
\text { de casos }\end{array}$ & Fracasos & Period & Endod & $\begin{array}{c}\text { Fractura } \\
\text { Radicular }\end{array}$ & Caries & Rest & Estudio \\
\hline 2-10 años & 45 & $3(6 \%)$ & 2 & 1 & - & - & - & Bergenholtz (29) \\
\hline 1-3,5 años & 34 & $1(3 \%)$ & 1 & - & - & - & - & Klavan (30) \\
\hline 5 años & 87 & 0 & - & - & - & - & - & Hamp (31) \\
\hline $1-7$ años & 34 & $7(21 \%)$ & 1 & 6 & - & - & - & Erpenstein (33) \\
\hline 5-13 años & 52 & 0 & - & - & - & - & - & Hou (25) \\
\hline 2-23 años & 49 & $4(8 \%)$ & - & 3 & - & 1 & - & Basten (36) \\
\hline 10 años & 146 & $32(22 \%)$ & 11 & 17 & 3 & - & 1 & Blomlöf(32) \\
\hline 3-11 años & 175 & $12(7 \%)$ & 3 & 4 & 2 & 3 & - & Carnevale (11) \\
\hline 10 años & 100 & $45(45 \%)$ & 10 & 7 & 25 & - & 3 & Langer (37) \\
\hline 10 años & 28 & $9(32 \%)$ & 2 & 5 & 1 & 1 & - & Buhler (38) \\
\hline
\end{tabular}




\section{COIMPARACIÓN DE LA RESECCIÓN RADICULAR CON OTRAS ALTERNATIVAS DE TRATAIMIENTO}

La eficacia de la RR para el tratamiento de los compromisos de furca es comparada con frecuencia con el abordaje abierto o con la regeneración tisular guiada (RTG). Pontoriero y Lindhe (40), diseñaron un ensayo clínico para evaluar efecto de la RTG en el tratamiento de furca grado II en molares superiores. Se incluyeron 28 pacientes entre 21 y 59 años de edad. Los autores demostraron que la RTG no es mejor que el debridamiento a colgajo abierto para el tratamiento de las furcas mesiales y distales de los molares superiores, probablemente debido a las dificultades de accesibilidad, la manipulación de los tejidos blandos y a la contaminación encontrada con los procedimientos de RTG en molares superiores. Los resultados de este estudio han sido corroborados por otros investigadores $(41,42)$. Debido a la posibilidad de fractura de los molares inferiores tratados con $R R(17,18,37$, 43), la RTG puede representar una alternativa razonable para compromisos de furca grado II en molares inferiores (44).

\section{CONCLUSIONES}

Los resultados de los estudios que evalúan la efectividad a largo plazo de la RR indican una tasa de éxito variable que va desde el 62 hasta el 100\%. La RR puede tratar compromisos de furca con características específicas que no pueden ser resueltos con otros enfoques quirúrgicos o no quirúrgicos. Para obtener resultados exitosos es fundamental comprender las particularidades anatómicas y morfológicas de las furcas y las limitaciones que estas presentan. La mayoría de fracasos informados no fueron de origen periodontal sino debidos a complicaciones endodónticas y fracturas radiculares. Por esta razón, los factores que pueden influir en el resultado de los procedimientos de $R R$ incluyen: la viabilidad en el tratamiento de los conductos radiculares, las fuerzas oclusales, la longitud del espacio edéntulo a restaurar, y la longitud, amplitud y forma de la raíz.

\section{BIBLIOGRAFÍA}

1. Al-Shammari KF, Kazor CE, Wang HL. Molar root anatomy and management of furcation defects. J Clin Periodontol $2001 ; 28: 730-40$.
2. DeSanctis M, Murphy KG. The role of resective periodontal surgery in the treatment of furcation defects. Periodontol 2000 2000; 22:154-68.

3. American Academy of Periodontology. Glossary of periodontal terms, $4^{\text {th }}$ edition. Chicago, Illinois. 2001.

4. Basaraba N. Root amputation and tooth hemisection. Dent Clin North Am 1969; 13:121-32.

5. Vandersall DC, Detamore RJ.The mandibular molar class III furcation invasion: a review of treatment options and a case report of tunneling. J Am Dent Assoc 2002;133: $55-60$.

6. Bohnenkamp DM, Garcia LT. Fixed restoration of sectioned mandibular molar teeth. Compend Contin Educ Dent 2004;25:920-4.

7. Lin CC, Tsai YL, Li UM, Chang YC, Lin CP, Jeng JH. Horizontal/oblique root fractures in the palatal root of maxillary molars with associated periodontal destruction: case reports. Int Endod J 2008;41:442-7.

8. Muzzi L, Nieri M, Cattabriga M, Rotundo R, Cairo F, Pini Prato GP. The potential prognostic value of some periodontal factors for tooth loss: a retrospective multilevel analysis on periodontal patients treated and maintained over 10 years. J Periodontol 2006;77: 2084-9.

9. Mullally B, Ziada H, Irwin C, Allen E, Byrne PJ. Periodontics: 7. Periodontal problems associated with compromised posterior teeth. Dent Update 2007;34: 607-10.

10. Svärdström G, Wennström JL. Periodontal treatment decisions for molars: an analysis of influencing factors and long-term outcome. J Periodontol 2000;71:579-85.

11. Carnevale G,Pontoriero R, di Febo G. Long-term effects of root-resective therapy in furcation-involved molars. A 10-year longitudinal study.J Clin Periodontol 1998;25: 209-14.

12. Goodacre CJ, Bernal G, Rungcharassaeng K, Kan JY. Clinical complications in fixed prosthodontics. J Prosthet Dent 2003;90:31-41.

13. Augereau D, Pierrisnard L, Renault P, Barquins M. Prosthetic restoration after coronoradicular resection: mechanical behavior of the distal root remaining and surrounding bone. J Prosthet Dent 1998;80:467-73. 
14. Hou GL, Tsai CC. Clinical significance of tooth morphology correlated with periodontal disease-I. Kaohsiung J Med Sci 1997;13:200-12.

15. Roussa E. Anatomic characteristics of the furcation and root surfaces of molar teeth and their significance in the clinical management of marginal periodontitis. Clin Anat 1998;11:177-86.

16. Guzy GE, Nichols JI. In vitro comparison of intact endodontically treated treated teeth with and without endo-post reinforcement. J Prosthet Dent 1979:42;39-42.

17. Abou-Rass M, Jann JM, Jobe D, Tsutsui F. Preparation of space for poting: Effect on thickness of canal walls and incidence of perforation in molars. J Am Dent Assoc 1982:104:834-37.

18. Torbjorner A, Karlsson S, Odman PA. Survival rate and failure characteristics for two post designs. J Prosthet Dent 1995:73:439-44.

19. Heydecke G, Peters MC. The restoration of endodontically treated, single-rooted teeth with cast or direct posts and cores: a systematic review.J Prosthet Dent 2002;87:380-6.

20. Mannocci F, Bertelli E, Sherriff M, Watson TF, Ford TR. Three-year clinical comparison of survival of endodontically treated teeth restored with either full cast coverage or with direct composite restoration. J Prosthet Dent 2002;88:297-301.

21. Fokkinga WA, Kreulen CM, Vallittu PK, Creugers NH. A structured analysis of in vitro failure loads and failure modes of fiber, metal, and ceramic post-and-core systems. Int J Prosthodont 2004;17:476-82.

22. Kutesa-Mutebi A, Osman YI. Effect of the ferrule on fracture resistance of teeth restored with prefabricated posts and composite cores. Afr Health Sci 2004;4:131-5.

23. Newell DH. The diagnosis and treatment of molar furcation invasions. Dent Clin North Am 1998;42:301-37.

24. Keough B. Occlusal considerations in periodontal prosthetics. Int J Periodontics Restorative Dent 1992; 12 : 359-71.

25. Hou GL, Tsai CC, Weisgold AS. Treatment of molar furcation involvement using root separation and a crown and sleeve-coping telescopic denture. A longitudinal study. J Periodontol 1999;70:1098-109.
26. Silverstein LH, Moskowitz ME, Kurtzman D, Faiella R, Shatz PC. Prosthetic considerations with periodontal root resective therapy, Part 1: Root amputations. Dent Today 1999;18:82-5.

27. Silverstein LH, Moskowitz ME, Kurtzman D, Shatz PC, Gornstein RA. Prosthetic considerations with periodontal root resective therapy, Part 2. Hemisections. Dent Today 1999;18:86-9.

28. Newell DH. The role of the prosthodontist in restoring root-resected molars: a study of 70 molar root resections. J Prosthet Dent 1991;65:7-15.

29. Bergenholtz A. Radectomy of multirooted teeth. J Am Dent Assoc 1972;85:870-5.

30. Klavan B. Clinical observations following root amputation in maxillary molar teeth. J Periodontol 1975; 46:1-5.

31. Hamp SE, Nyman S, Lindhe J. Periodontal treatment of multirooted teeth. Results after 5 years. J Clin Periodontol 1975;2:126-35.

32. Blomlöf L, Jansson L, Appelgren R, Ehnevid H, Lindskog S. Prognosis and mortality of root-resected molars. Int J Periodontics Restorative Dent 1997;17:190-201.

33. Erpenstein H. A 3-year study of hemisectioned molars. J Clin Periodontol 1983;10:1-10.

34. Silness J, Löe H. Periodontal disease in pregnancy 3. Response to local treatment. Acta Odontol Scand 1966; 24:747-59.

35. Loe H, Silness J. Periodontal disease in pregnancy 1. Prevalence and severity. Acta Odontol Scand 1963;21: 533-51.

36. Basten CH, Ammons WF Jr, Persson R. Long-term evaluation of root-resected molars: a retrospective study. Int J Periodontics Restorative Dent 1996;16:206-19.

37. Langer B, Stein SD, Wagenberg B. An evaluation of root resections. A ten-year study. J Periodontol 1981:52:71922.

38. Bühler H. Evaluation of root-resected teeth. Results after 10 years. J Periodontol 1988;59:805-10.

39. Buhler HB. Survival rates of hemisected teeth: an attempt to compare them with survival rates of alloplastic 
implants. Int J Periodontics Restorative Dent 1994;14: 537-43.

40. Pontoriero $R$, Lindhe J. Guided tissue regeneration in the treatment of degree II furcations in maxillary molars. J Clin Periodontol 1995;22:756-63.

41. Avera JB, Camargo PM, Klokkevold PR, Kenney EB, Lekovic V. Guided tissue regeneration in Class II furcation involved maxillary molars: a controlled study of 8 split-mouth cases. J Periodontol 1998; 69:1020-6.

42. Metzler DG, Seamons BC, Mellonig JT, Gher ME, Gray $\mathrm{JL}$. Clinical evaluation of guided tissue regeneration in the treatment of maxillary class II molar furcation invasions. J Periodontol 1991;62:353-60.
43. Little L, Beck F, Bagri B, Horton H. Lack of furcal bone loss following the tunneling procedure. J Clin Periodontol 1995;22:637-41.

44. Schallhorn RG, McClain PK. Combined osseous composite grafting, root conditioning, and guided tissue regeneration. Int J Periodontics Restorative Dent 1988; 8:9-31.

\section{CORRESPONDENCIA}

Carlos Martín Ardila Medina

Carrera 47 No. 20 sur 46 Envigado Antioquia 57(4) 3348122

cmartin@odontologia.udea.edu.co 